\title{
Data Management Issues Highlighted in TMS/ASM Workshop Report
}

\author{
Lynne Robinson
}

Defining the data challenges associated with building the materials innovation infrastructure at the core of the U.S. Materials Genome Initiative (MGI) was the focus of an intersociety scoping session organized by TMS and ASM International during the Materials Science \& Technology 2012 Conference (MS\&T'12). The effort drew on the perspectives of members from a wide range of professional societies representing the diversity of materials science and engineering (MSE) disciplines Their collective insights have been compiled into a report recently released by TMS and ASM, and available at http://materialsinnovation.tms .org/2012IntersocietyScopingSession/.

"This report represents an important step forward in the conversation surrounding interdisciplinary collaboration, as it identifies the primary categories of data associated with MSEparticularly from the structural materials perspective," said George Spanos, TMS Technical Director. "It also explores the degree to which these categories of data are being actively shared across fields, and considers some key data management issues."

The 2012 event built on the momentum of an intersociety MGI scoping session convened by TMS at MS\&T'11, and was planned in coordination with an MGI workshop led by the National Institute of Standards and Technology (NIST) in May 2012. The 2011 scoping session explored broad strategies that the MSE community could undertake to enhance collaboration, while the NIST-led workshop outlined key barriers to establishing the digital data component of a materials innovation infrastructure. (The report of the 2011 scop- ing session can be downloaded at http ://materialsinnovation.tms.org/docs $/ p d f$ s/2011MGIScopingSession.pdf and the summary report of the NIST

\section{Table I. Primary Categories of Materials Data}

*Acoustical Data

*Aesthetic Data

Atomic Potential Data

Composition Data

Constituent Material Data

Cost Data

Dynamic Mechanical Property Data (e.g., viscosity)

Electrical Data

End-Use Mechanical Property Data

Environmental/Health/Safety Data (e.g., legislation)

Failure Data (wear/fracture/corrosion)

Fundamental Mechanical Property Data

Kinetic Data

*Magnetic Data

Manufacturing Performance Data (e.g., materials fidelity)

*Materials Emission Data (e.g., photons, electrons)

Materials Meta-data

Microstructure Data

Mobility Data (e.g., diffusion)

*Nuclear property data

Optical Data

Oxidation/Corrosion Data

Phases Data

Processing Data

*Qualitative Data

*Statistical Data

Surface Morphology Data (e.g., roughness)

Thermodynamic Data

Thermophysical Data

*Validation Data

* Categories marked with an asterisk were not further rated for metrics (see Table II) because discussion time was limited and the workshop participants decided to focus in the time allotted on the categories without asterisks. workshop is available at http://events. energetics.com/MGIWorkshop/pdfs /MGI-Workshop-Report-FINAL.pdf.)

The MGI, announced in June 2011 by President Barack Obama, is a multiagency initiative designed to create a new era of resources, infrastructure, and policy that support U.S. institutions in discovering, manufacturing, and deploying advanced materials at an accelerated pace with significantly reduced costs. Key enablers of the MGI materials innovation infrastructure are the development of advanced computational and experimental tools, as well as the management of digital data across platforms and organizations. This ambitious undertaking will only be possible, noted Spanos, through the cooperative efforts of four foundational groups - government, industry, and academia, with professional societies playing a leading role in fostering the necessary climate of collaboration.

"A core tenet of the MGI is that cross-organizational and interdisciplinary collaboration will yield better results than isolated efforts," said Spanos. "The TMS/ASM collaboration is an excellent example of that. It allows these two organizations to utilize their respective strengths in a coordinated manner to make faster progress towards building the materials innovation infrastructure. Additionally, bringing together their networks of professional society contacts models the collaboration called for by the MGI. This can then be extended to the members of these organizations to encourage similar approaches."

Based on a recommendation in the 2011 scoping session report to "develop a language and taxonomy for 
Table II. Types/Varieties of Data

\section{Raw Data}

Metadata (descriptors about the raw data)

Derived Data

Method (used to obtain the data)

Model Verification and Validation Data

Design Values

Statistical Data

data collection and sharing," the 2012 scoping session focused on identifying primary categories of data associated with MSE-with an emphasis on structural materials - and assessing the current states of sharing primary data and tools. "The report presents a list of data categories (Table I) that scientists and engineers should be aware of when they are seeking to work with and understand structural materials data," said Spanos. "It also provides background information on these categories (Table II), including an assessment of the maturity of data sharing efforts in these areas. This is important information to individuals and organizations who wish to fill in the gaps, in terms of data sharing efforts, or to start new initiatives."

To determine the current states of data sharing and tools to develop such data, workshop participants rated each identified data category in terms of the MSE community's familiarity with the data, the data's accessibility, and the readiness of the data and tools for commercial implementation. In general, it was determined that high familiarity with data doesn't necessarily correlate with the level of maturity or

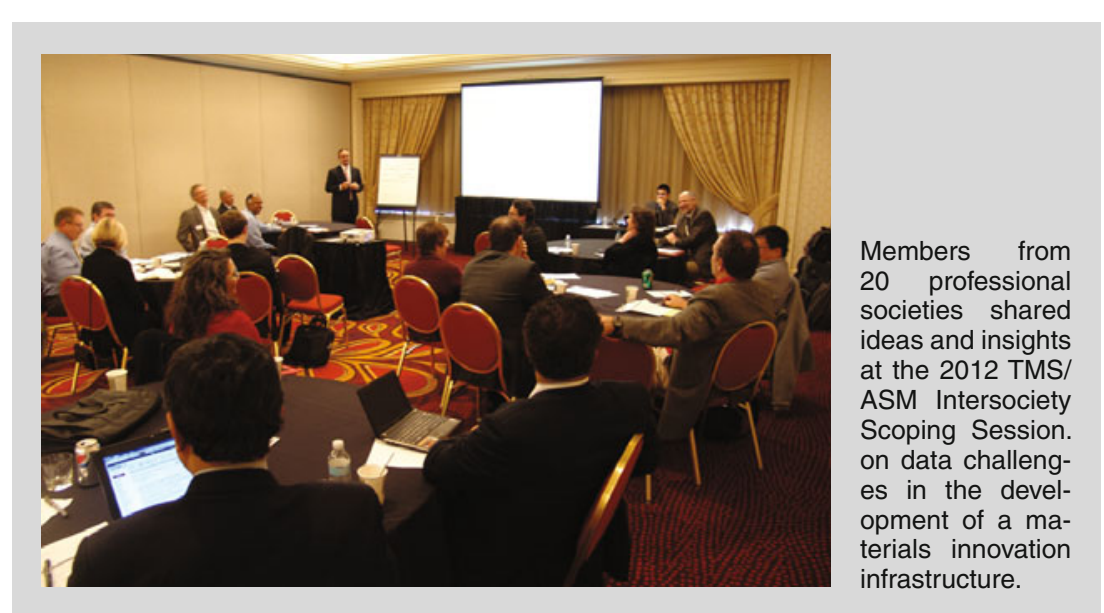

accessibility of the data. However, the reverse situation - low familiarity and high accessibility or maturity — was not observed.

"An example of one of the key issues brought up in the workshop was that there are vast amounts of mature, useful data that are not available to the community because of their form of storage," said David Howe, TMS

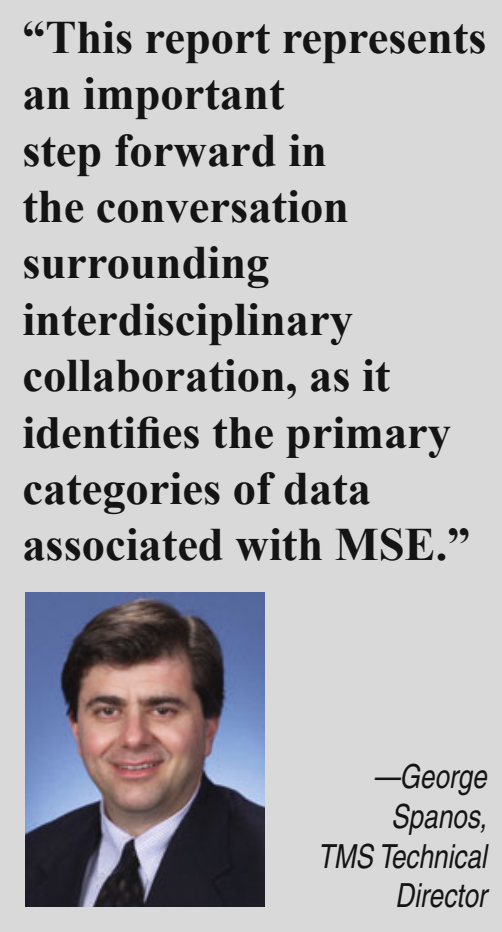

Technical Specialist. "That is to say, older data that is stored only on paper is not accessible, yet could be very useful if made available. A challenge for the field will be to make these older data sets available to an increased degree." infrastructure.
Spanos noted that the information presented in the 2012 scoping session report is intended to catalyze broader efforts across MSE disciplines. "Workshop participants can use this report to inform colleagues and other contacts about the benefits and challenges associated with the call for increased data sharing, standardization, and collaboration, as well as develop strategies for contributing to these efforts," he said. He added that TMS specifically plans to build and expand on the results of the report as it progresses toward its strategic goal of "being the recognized home for and advocate of materials and manufacturing innovation."

"In addition to developing resources and venues for convening the MSE community on these topics," Spanos said, "TMS will use the report's insights to inform development of existing resources for data sharing and collaboration." These TMS projects include continued enhancement of the Cyberinfrastructure Portal (materialsinnovation.tms.org/portal) and 3D Materials Atlas (materialsinnovation .tms.org/materialsatlas), as well as the new MGI Digital Data Community, a collaborative project with NIST that will serve as a forum for MSE practitioners to interact, share information, and build collaborations.

Bolstering these efforts, said Spanos, is the continued collaboration of professional societies, including TMS and ASM, in convening the field and developing resources to overcome barriers to collaboration. "As the MGI continues to stress the importance of data sharing and collaboration across different fields, it is critical to initiate the interdisciplinary conversations needed to make this happen," he said. "The 2012 scoping session report contains the collective input of scientists, engineers, and data experts from various disciplines and membership in some 20 different professional societies. The report is both an example of the needed collaboration among these experts, as well as a resource that identifies and maps some of the key data management issues within MSE and its allied fields."

Lynne Robinson is a news and feature writer for TMS. 